\title{
Nondestructive Control \\ of Physicomechanical Properties and Quality of Graphitized Carbon Products
}

Elena Z. Kovarskaya, Igor B. Moskovenko and Maria S. Shadrina* «ZVUK»Ltd 17 Beloostrovskaya Str., Saint-Petersburg, 197342, Russia

Received 28.02.2018, received in revised form 04.03.2018, accepted 29.04.2018

The opportunity and expediency of use of the acoustic quality monitoring based on measurement of natural oscillation frequencies of articles, for an estimation of physicomechanical properties and quality the bottom blocks burnt and not burnt ("green") anodes of electrolyzers is displayed. The results of acoustic monitoring expressed in sound indexes, can be effectively used at prediction of working capacity of carbon articles used by manufacture of aluminium.

Keywords: carbon materials, nondestructive control, physicomechanical properties, acoustic control methods, frequencies of natural oscillations.

Citation: Kovarskaya E.Z., Moskovenko I.B., Shadrina M.S. Nondestructive control of physicomechanical properties and quality of graphitized carbon products, J. Sib. Fed. Univ. Eng. technol., 2018, 11(4), 443-452. DOI: 10.17516/1999-494X-0067.

\section{Неразрушающий контроль} физико-механических свойств

\section{и качества углеграфитовых изделий}

\author{
Е.3. Коварская, И.Б. Московенко, М.С. Шадрина \\ $O O O « 3 В У K »$
}

Россия, 197342, Санкт-Петербург, ул. Белоостровская, 17

\begin{abstract}
Показана возможность и иелесообразность использования акустических методов контроля, основанных на измерении частот собственных колебаний изделий, для оценки физикомеханических свойств и качества подовых блоков, обожженных и необожженных («зеленых») анодов электролизеров. Результаты акустического контроля, выраженные в звуковых индексах, могут быть эффективно применены при прогнозировании работоспособности углеродных изделий, используемых при производстве алюминия.
\end{abstract}

(C) Siberian Federal University. All rights reserved

* Corresponding author E-mail address: info@ndtest.ru 
Ключевые слова: углеродные материалы, неразрушающий контроль, физико-механические свойства, акустические методы контроля, частоты собственных колебаний.

Современный уровень технологии изготовления углеграфитовых изделий предусматривает необходимость контроля их физико-механических свойств, который осуществляется, как правило, разрушающими методами на образцах, которые вырезаются из изделий, отобранных из партии. В условиях действующего производства такой метод контроля не может служить надежной и адекватной оценкой технологической партии изделий; кроме того, он достаточно низкопроизводительный. Использование низкочастотного акустического метода, основанного на измерении частот собственных колебаний изделий, позволяет, с одной стороны, обеспечить возможность определения физико-механических свойств реальных изделий без их разрушения, с другой - существенно повысить надежность и производительность контроля.

Способ неразрушающего акустического контроля физико-механических свойств применительно к контролю углеродных изделий может эффективно применяться в электродной промышленности при производстве углеродных изделий, а также в отраслях, потребляющих эти изделия, в таких как черная и цветная металлургия, химическая и абразивная промышленность и др. Возможности метода могут быть рассмотрены на примере его применения для оценки физико-механических свойств и качества углеродных изделий, используемых при производстве алюминия. Еще в 90-х гг. прошлого века в результате совместной работы Всесоюзного научно-исследовательского и проектного института ВАМИ и Всесоюзного научно-исследовательского института абразивов и шлифования (ВНИИАШ) была показана возможность и целесообразность использования низкочастотного акустического метода контроля для оценки физико-механических свойств подовых блоков и обожженных анодов электролизеров при производстве алюминия. На Новосибирском электродном заводе и Саяногорском алюминиевом заводе выполнялись измерения с помощью более ранней модификации выпускаемых на данный момент фирмой «ЗВУК» приборов - прибором «Звук-203». Действие прибора основано на методе свободных колебаний, возбуждаемых в изделии ударом. За прошедшее время сменилось, по крайней мере, два поколения приборов типа «Звук». В настоящее время выпускаемые ООО «ЗВУК» приборы «Звук-203М» и «Звук-130» прошли государственные испытания, сертифицированы, включены в Государственный реестр средств измерений, достаточно широко эксплуатируются в нашей стране и поставляются на экспорт в развитые зарубежные страны.

Определение физико-механических характеристик материала акустическим методом основано на связи частот собственных колебаний (ЧСК) изделий со скоростью распространения акустических волн в материале $(\mathrm{Cl})$, из которого они изготовлены. Параметр $\mathrm{Cl}$ имеет физический смысл скорости распространения продольных упругих колебаний в бесконечно длинном стержне, изготовленном из того же материала, что и материал контролируемого изделия, и связан с модулем нормальной упругости Е и плотностью материала изделия $\rho$ известным соотношением (1):

$$
\mathrm{C}_{l}=\sqrt{\frac{E}{\rho}}
$$


Скорость распространения акустических волн, или скорость звука, связана с ЧСК изделий следующим соотношением (2):

$$
f_{i}=F_{i} \times C_{l},
$$

где $f_{i}$ - ЧСК изделия, соответствующая определенному виду колебаний (продольным, изгибным, плоским и т.д.); $F_{i}$ - коэффициент формы, определяемый размерами и формой контролируемого изделия, а также видом возбуждаемых в нем колебаний i.

Нашими исследованиями, а также исследованиями других авторов [1] убедительно показано, что в качестве меры физико-механических свойств различного рода изделий при проведении акустического контроля целесообразно принять параметр $\mathrm{Cl}$, пронормированный в так называемых звуковых индексах (ЗИ). Для характеристики свойств углеродных блоков показатель ЗИ в значительной степени определяется пористостью контролируемых изделий.

Звуковой индекс - это интервал приведенной скорости распространения акустических волн в изделии, в т.ч. в углеродном блоке. Звуковой индекс обозначается целым нечетным числом, равным среднему в данной градации значению параметра $\mathrm{Cl}$, выраженному в м/с и умноженному на $10^{-2}(3)$ :

$$
\text { ЗИ }=\frac{C_{l} \max +C_{l} \min }{2} \times 10^{-2} .
$$

В табл. 1 приводятся значения параметра $\mathrm{Cl}$, соответствующие звуковым индексам от 17 до 35, характеризующие физико-механические свойства основных видов углеродных изделий, используемых при производстве алюминия.

Указанные ЗИ с достаточной степенью точности характеризуют физико-механические свойства углеродных изделий и могут быть применены для подбора комплектов изделий со стабильными, заранее заданными свойствами. Так как при использовании комплексного показателя ЗИ свойства определяются не на образцах, а сразу во всем изделии, существенно повышается надежность контроля, которая с учетом большой экспрессности неразрушающего контроля (до 20 с) позволяет осуществлять сплошной контроль углеродных изделий.

Следует отметить, что параметр $\mathrm{Cl}$ является высокоинформативным параметром и в ряде случаев позволяет достаточно полно судить о физико-механических свойствах (определяют работоспособность изделий из различных материалов, начиная с полимерных и кончая твердыми и сверхтвердыми материалами, причем для последних этот параметр позволяет получать

Таблица 1. Соотношение между звуковыми индексами (ЗИ) и скоростью звука $\mathrm{Cl}$ Table 1. The relationship between the sound indexes (SI) and the acoustic wave velocity $\mathrm{Cl}$

\begin{tabular}{|c|c|}
\hline Звуковой индекс (ЗИ) & Диапазон значений Cl, м/c \\
\hline 17 & от 1600 до 1800 \\
\hline 19 & свыше 1800 до 2000 \\
\hline$\ldots$ & $\ldots$ \\
\hline 33 & свыше 3200 до 3400 \\
\hline 35 & свыше 3400 до 3600 \\
\hline
\end{tabular}

$$
-445-
$$


Таблица 2. Физико-механические свойства некоторых материалов

Table 2. Physicomechanical properties of some materials

\begin{tabular}{|c|c|c|c|c|}
\hline Материал & $\mathrm{Cl}, \mathrm{M} / \mathrm{c}$ & ЗИ & Е, ГПа 10 & Н, ГПа $\cdot 10^{-2}$ \\
\hline Алмаз & $15700-15900$ & $157-159$ & $50,2-85,0$ & 10000 \\
\hline Эльбор & $12000-15600$ & $125-157$ & $62,0-95,0$ & 9000 \\
\hline Карбид бора & 10800 & $107-109$ & 29,6 & $3700-4300$ \\
\hline Термокорунд & $9600-10200$ & $97-101$ & $35,5-41,0$ & $2000-2400$ \\
\hline Карбид вольфрама & 6700 & 67 & 72,2 & 1730 \\
\hline Сталь & $4900-5200$ & $49-51$ & - & - \\
\hline Алюминий & $5100-5200$ & 51 & - & - \\
\hline Чугун & $3900-4300$ & $39-43$ & - & - \\
\hline Медь & $3400-3850$ & $35-39$ & - & - \\
\hline Плавиковый шпат & 2140 & 21 & - & - \\
\hline Кальцит & 1690 & 17 & - & - \\
\hline Графит & $800-1100$ & $9-11$ & - & - \\
\hline Тефлон & 430 & 5 & - & - \\
\hline Полиэтилен & 390 & 3 & - & - \\
\hline
\end{tabular}

более достоверную информацию о такой важной характеристике подобных материалов, как микротвердость), чем обычно используемый для оценки физико-механических свойств таких материалов модуль нормальной упругости E [1].

В табл. 2 приведены значения параметра $\mathrm{Cl}$ и ЗИ для некоторых видов материалов, определенные акустическим методом с применением измерителей типа «Звук» или рассчитанные по известным значениям Е и $\rho$ В этой же таблице для некоторых твердых и сверхтвердых материалов для сравнения приведены значения модуля упругости Е и микротвердости $\mathrm{H}$.

Как следует из данных табл. 2, с помощью скорости звука $\mathrm{Cl}$ могут оцениваться физикомеханические свойства изделий из самых разнообразных материалов, причем для твердых и сверхтвердых материалов с ростом их микротвердости значения $\mathrm{Cl}$ соответственно возрастают, в отличие от обычного применяемого для оценки свойств подобных изделий модуля упругости Е, для которого такая однозначная зависимость отсутствует. Приведенные данные позволили предположить наличие аналогичных зависимостей между физико-механическими свойствами углеродных изделий и скоростью $\mathrm{Cl}$.

Работами, проведенными на ряде алюминиевых и электродных заводов в различные периоды времени, было установлено, что физико-механические свойства подовых блоков значительно различаются даже у одного изготовителя [2]. Тем не менее опыт эксплуатации промышленных электролизеров демонстрирует, что для надежной гарантии работоспособности подины необходимо проводить ее монтаж из блоков одинакового качества, при этом наилучших технико-экономических показателей в работе электролизера, в том числе и по сроку службы, можно достичь при определенных физико-механических свойствах углеродных блоков. При низких прочностных свойствах (им соответствуют меньшие значения ЗИ) блоки подвержены в большей мере сколам, трещинам (даже при чисто механических ударах, нагрузках еще в про- 
цессе их транспортировки и монтажа электролизных ванн). При больших значениях ЗИ блоки имеют повышенную механическую прочность, но при этом могут проявляться хрупкость и, в процессе эксплуатации, внутренние напряжения из-за капиллярного пропитывания электролитом блоков с пониженным значением пористости (значения ЗИ с уменьшением пористости возрастают), что может привести к преждевременному образованию трещин.

В соответствии с рекомендациями ВАМИ на Новосибирском электродном заводе был освоен выпуск блоков подовых с нормированными физико-механическими свойствами в соответствии со специально разработанными ТУ 48-4804-27-91, предусматривающими маркировку на блоках результатов акустического контроля в виде звуковых индексов. Такие блоки по запросу заводов, производящих алюминий, поставлялись по несколько повышенной цене, и результаты акустического контроля, замаркированные на блоках, учитывались при подборе комплектов, используемых для монтажа электролизеров. В дальнейшем по причинам в основном организационного характера эти работы применительно к контролю подобных углеродных изделий были практически прекращены. Однако применительно к другим видам изделий, в первую очередь различного рода абразивным инструментам, эти работы продолжались, и в 2007 г. был разработан и введен в действие ГОСТ Р 52710 - 2007 «Инструмент абразивный. Акустический метод определения твердости и звуковых индексов по скорости распространения акустических волн», в соответствии с которым в настоящее время производится подобный контроль на ряде фирм производителей и потребителей абразивной продукции. Этот ГОСТ может быть положен в основу нормативно-технической документации или стандарта, обеспечивающих возможность аналогичного акустического контроля углеродной продукции.

В настоящее время в алюминиевой промышленности вместо маркировки на блоках результатов акустического контроля реализованы несколько иные принципы комплектации. В частности, с 2014-2015 гг. на алюминиевых заводах начаты работы по комплектации подин подовыми блоками с близкими характеристиками. В качестве базовых параметров катодных блоков приняты величина удельного электросопротивления (УЭС) и механическая прочность на сжатие. Причем при проведении разрушающих испытаний используется минимальное количество кернов, которое не является достаточным для оценки неоднородности анодного блока и всей партии в целом. По нашему мнению, взамен определения механической прочности на образцах более экономично и эффективно может быть взята известная зависимость предела прочности от скорости $\mathrm{Cl}$.

Скорость распространения акустических волн $\mathrm{Cl}$ (скорость звука) связана известным соотношением с важными параметрами, характеризующими физико-механические свойства материала углеродных блоков, - модулем нормальной упругости (модуль Юнга) Е и плотностью материала $\rho$. В свою очередь, скорость звука в углеродных изделиях связана корреляционной зависимостью с его прочностными, структурными и другими физико-механическими и электрофизическими характеристиками (в частности, установлена тесная корреляционная связь между скоростью звука, определенной ультразвуковым методом, прочностью, пористостью, объемной плотностью и удельным электросопротивлением углеродных изделий) (рис. 1) [3, 4].

Для различных материалов, в т. ч. углеродных, неоднократно было экспериментально показано наличие тесной линейной корреляционной связи между параметрами $\mathrm{Cl}$ и скоростью звука, определяемой ультразвуковым методом $\mathrm{C}$, с коэффициентом корреляции 

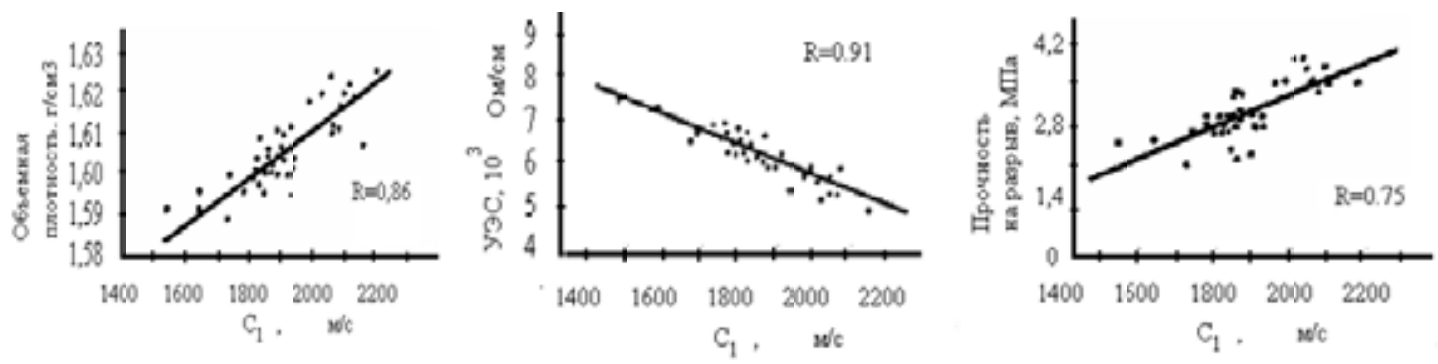

Рис. 1. Корреляционные зависимости между скоростью звука $\mathrm{Cl}$ и физико-механическими свойствами подовых блоков электролизеров для производства алюминия: объемной плотностью, удельным электросопротивлением и прочностью на разрыв

Fig. 1. Correlation between the acoustic wave velocity $\mathrm{Cl}$ and the physicomechanical properties of the hearthlevel blocks of baths of electrolyzers used by manufacture of aluminium: bulk density, resistivity and tensile strength

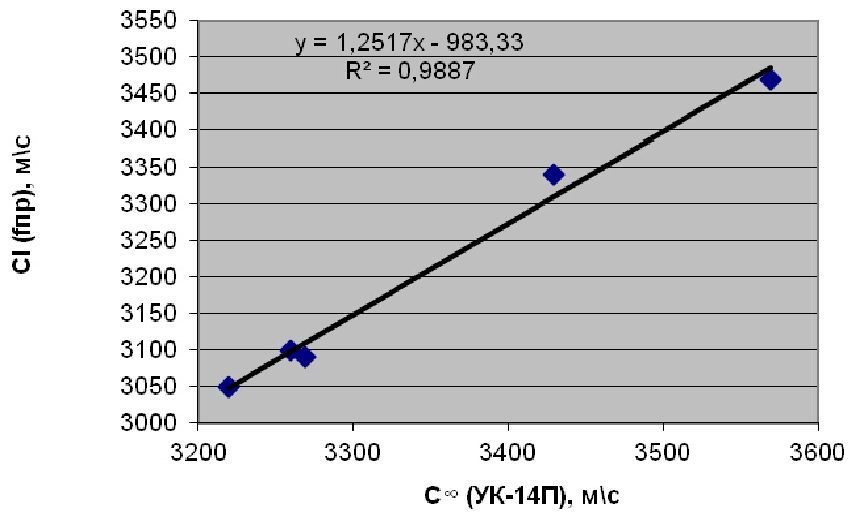

Рис. 2. Определение физико-механических свойств образцов из графитового материала в форме стержня с размерами $40 \times 40 \times 120$ мм. $\mathrm{R}=0,99$

Fig. 2. Determination of physicomechanical properties of samples from graphite material in the form of a rod with dimensions of $40 \times 40 \times 120 \mathrm{~mm} . \mathrm{R}=0.99$

$\mathrm{R}=0,90$ и выше. Пример такой зависимости приведен на рис. 2. Это позволяет равноценно использовать оба параметра для дальнейшего определения физико-механических свойств контролируемых изделий. Как известно, скорость распространения акустических волн $C_{l}=\sqrt{\frac{E}{\rho}}$ связана со скоростью звука, определяемой ультразвуковым методом С $\infty$, известным соотношением:

$$
\mathrm{c}_{\infty} / \mathrm{C}_{l}=\sqrt{\frac{(1-v)}{(1+v)(1-2 v)}}=1,054
$$

где $v$ - коэффициент Пуассона, который для углеродных изделий с достаточной степенью точности может быть принят равным $v=0,2$.

Также необходимо отметить, что рядом зарубежных фирм модуль нормальной упругости включается в число основных показателей, характеризующих физико-механические свойства углеродных блоков. 
В 2016 году на случайно отобранных образцах катодных блоков в условиях Новосибирского электродного завода были проведены аналогичные испытания по оценке возможности определения физико-механических свойств и дефектоскопии образцов катодных блоков с помощью прибора «Звук-203М». В качестве объектов контроля были предоставлены шесть блоков из различных технологических партий, в т. ч. три блока приняты ОТК как годные, а три блока забракованы по результатам визуального контроля по наличию трещин и рыхлой структуры, вскрывшихся при обработке желоба. Также измерения проводились при различных условиях складирования.

На рис. 3 приведены зависимости между различными характеристиками физикомеханических свойств, полученными для указанных блоков. Там же указаны коэффициенты корреляции для линейной зависимости между этими характеристиками.

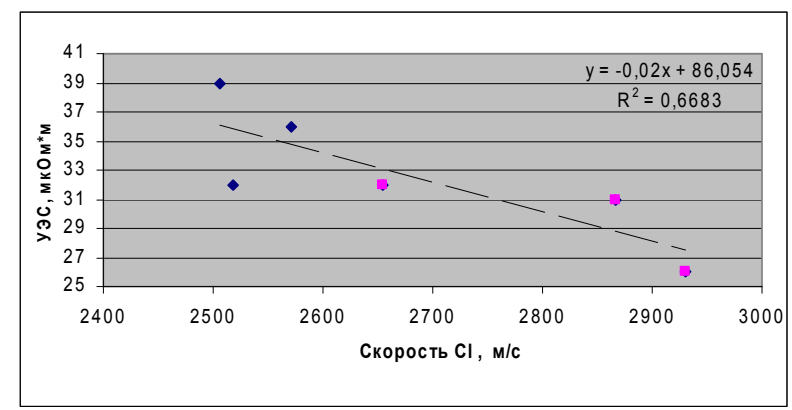

а) коэффициент корреляции $\mathrm{R}=0,82$

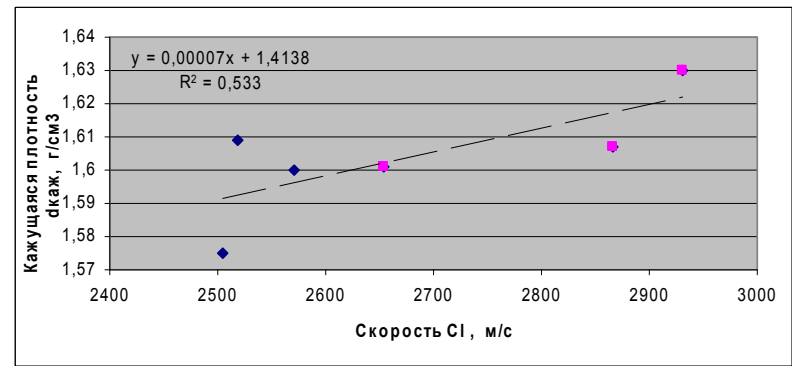

б) коэффициент корреляции $\mathrm{R}=0,73$

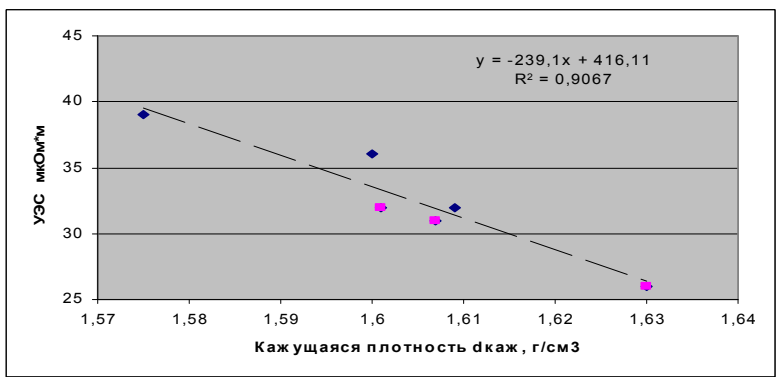

в) коэффициент корреляции $\mathrm{R}=0,95$

Рис. 3. Зависимости между физико-механическими свойствами тестовых катодных блоков: а) «УЭСскорость Сl»; б) «dкаж - скорость Сl»; в) «УЭС-дкаж»

Fig. 3. Dependences between physicomechanical properties of test cathode blocks: a) "resistivity - velocity Cl"; б) "bulk density - velocity Cl"; в) "resistivity - bulk density" 
Из представленных данных (рис. $3 a$ и 36 ) следует, что даже на такой малой и случайной выборке подтверждается характер ранее установленных зависимостей между результатами акустического контроля $\mathrm{Cl}$ (ЗИ) и результатами определения кажущейся плотности и удельного электросопротивления по принятым на предприятии методикам. Прибор «Звук-203М» позволяет проводить измерение ЧСК различных типов с достаточно высокой сходимостью и воспроизводимостью результатов измерений. Для изделий, забракованных ОТК, получены значения ЗИ $25(\mathrm{Cl}=2510-2570$ м/с), для годных - более высокие значения - ЗИ 27-29 $(\mathrm{Cl}=2650-2930 \mathrm{M} / \mathrm{c})$.

Таким образом, проведенное опробование подтверждает все ранее полученные результаты, показывает возможность использования приборов нового поколения «Звук-203М» для оперативного экспресс-контроля физико-механических свойств (качества) подовых блоков, в т. ч. до окончательной механической обработки, а также при складировании заготовок в несколько рядов.

В условиях ОАО «РУСАЛ Красноярский алюминиевый завод» было проведено определение физико-механических свойств образцов анодных блоков с применением измерителя частот собственных колебаний «Звук-203М». В качестве объектов контроля были предоставлены анодные блоки четырех производителей (три китайских, один российский). На рис. 4 даны результаты акустического контроля измеренных комплектов блоков в виде распределения по звуковым индексам. Значения ЗИ всех комплектов лежат в интервале от ЗИ 21 до ЗИ 25. Наибольшее различие физико-механических свойств блоков, выраженных в ЗИ, выявлено у комплекта производителя 4 и составляет три звуковых индекса. Наиболее равномерны по свойствам анодные блоки поставщиков 1 и 2 , имеющие один звуковой индекс ЗИ 23. Анодные блоки производителя 3 распределились по двум звуковым индексам, причем большая часть блоков имеет минимальный из распределения индекс ЗИ 21, что может свидетельствовать об ухудшении физико-механических характеристик данных образцов, например прочности, по сравнению с анодными блоками других представленных производителей. Анодные блоки этого производителя были отбракованы по нескольким низким показателям.

В соответствии с заданной программой испытаний из каждой выборки были отобраны блоки с минимальным и максимальным значением $\mathrm{Cl}$, а в выборке поставщика 4 - еще 2 блока с промежуточными значениями $\mathrm{Cl}$. От каждого из выбранных блоков было отобрано по одному

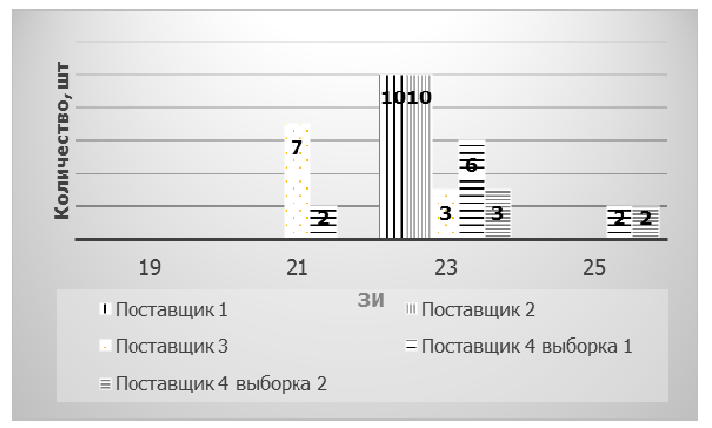

Рис. 4. Акустический контроль четырех партий анодов различных производителей

Fig. 4. Acoustic control of four batches of burnt anodes of various manufacturers 
керну, на которых были проведены определения физико-механических характеристик по методикам, принятым на предприятии. Образцы (керны), предназначенные для определения УЭС, были после определения УЭС дополнительно проконтролированы акустическим методом.

Из результатов предварительного эксперимента следует, что значения $\mathrm{Cl}$ кернов и блоков могут отличаться от 2 до $8 \%$, что соответствует переходу в соседний ЗИ.

Для получения данных, позволяющих устанавливать зависимости между результатами различных видов испытаний, следует оценивать реальный максимальный диапазон изменения $\mathrm{Cl}$ на репрезентативной выборке изделий неразрушающим экспресс-методом, а затем проводить измерения физико-механических свойств на образцах в трех - пяти точках диапазона $\mathrm{Cl}$ не менее, чем на трех образцах для каждой точки. При проведении разрушающих испытаний в такой серии следует использовать количество кернов, достаточное для оценки неоднородности анодного блока (минимально три штуки).

Выполненные работы подтвердили ранее установленные возможность и целесообразность оценки физико-механических свойств и качества анодных блоков с использованием акустических методов, основанных на измерении собственных частот с применением современных измерителей ЧСК типа «Звук-203М». Для построения соответствующих зависимостей и определения браковочных показателей следует проводить накопление статистических данных и сопоставление с разрушающими испытаниями на репрезентативной выборке изделий.

Также в условиях КрАЗа производилось определение физико-механических свойств образцов катодных блоков с применением того же измерителя частот собственных колебаний «Звук-203М». В качестве объектов контроля были предоставлены три комплекта блоков из двух технологических партий (15 шт. блоков в каждом комплекте). Все блоки приняты ОТК поставщика как годные и собраны в комплекты для монтажа электролизеров.

На рис. 5 представлены результаты акустического контроля трех комплектов блоков в виде распределения по звуковым индексам. Значения ЗИ всех комплектов лежат в интервале от ЗИ 25 до ЗИ 29. Различие физико-механических свойств блоков, выраженных в ЗИ, внутри каждого комплекта из обеих партий не превышает двух звуковых индексов.

С учетом полученного распределения была дана рекомендация не устанавливать рядом блоки с максимально отличающимися характеристиками, в нашем случае с ЗИ 25 и ЗИ 29.

При проведении контроля физико-механических свойств углеродных блоков акустическим методом результаты измерения частот собственных колебаний отличаются высокой

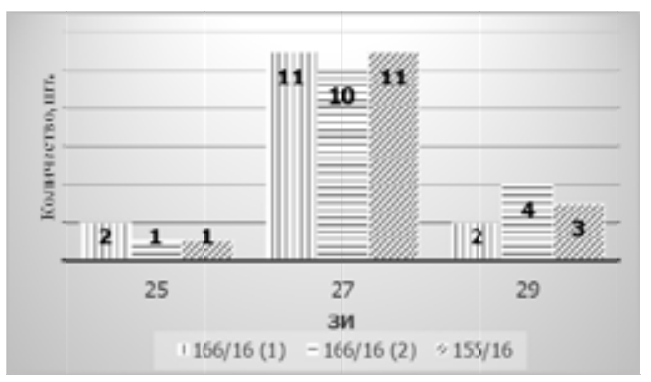

Рис. 5. Акустический контроль трех комплектов катодов

Fig. 5. Acoustic control of three sets of cathodes

$$
-451-
$$


стабильностью и воспроизводимостью, метод является бесконтактным и в отличие от других неразрушающих методов контроля, например ультразвуковых, не зависит от состояния наружной поверхности углеродного блока. Стабильность результатов контроля в ряде случаев позволяет получать информацию о наличии скрытых дефектов типа трещин, расслоений, раковин и т.П., о присутствии которых судят по значительным различиям результатов контроля при повторных измерениях. Отсутствие непосредственного контакта приемоизлучающей системы с углеродным блоком в момент измерения значительно упрощает сам процесс контроля, повышает его экспрессность и создает благоприятные условия для использования этого метода в автоматизированном производстве.

Сплошной (100 \%) неразрушающий акустический контроль качества углеродных блоков обеспечит идентичность и стабильность свойств углеродных изделий, применяемых при монтаже и ремонте электролизеров, может быть использован для контроля за соблюдением технологического процесса их производства, исключит попадание на монтаж электролизеров углеродных блоков с характеристиками, отличными от заданных, выявит причины нестабильности в технологии электролиза.

Контроль может быть рекомендован для экспресс-определения дефектных изделий и для применения у изготовителя как сплошной или выборочный контроль технологии, так и экспресс-контроль для выбора изделий при арбитражных испытаниях.

\section{Список литературы}

[1] Grebjonkin A.F., Krjukowskij W.A., Glagowskij B.A., Lyssanow W.S. und Moskowenko I.B. Zerstörungsfreie akustische Materialprüfung an Kohlenstoffanoden, Aluminium-66. Jahrgang. 1990, (9), 854-857.

[2] Гребенкин А.Ф., Крюковский В.А., Московенко И.Б. Исследование физико-механических свойств углеродных блоков различных изготовителей с помощью акустического метода. Решение экологических проблем в производстве алюминия: Сб. науч. тр. ВАМИ, Л.: ВАМИ, 1990, 62-72 [Grebyonkin A.F., Kryukovskiy V.A., Moskovenko I.B. Research of physicomechanical properties of carbon blocks of various manufacturers by means of an acoustic method. The solution of environmental problems in aluminum production: Collection of scientific papers VAMI, Leningrad: VAMI, 1990, 62-72 (in Russian)]

[3] Гребенкин А.Ф., Московенко И.Б., Савинов В.И., Ломоносов Л.В., Кузнецов Г.П., Глаговский Б.А. Неразрушающие методы контроля для оценки качества крупногабаритных углеродных изделий. Повышение эффективности и надежности работы алюминиевых электролизеров: Сб. Науч. тр. ВАМИ, Л.: ВАМИ, 1987, 26-31. [Grebyonkin A.F., Moskovenko I.B., Savinov V.I., Lomonosov L.V., Kuznetsov G.P., Glagovskiy B.A. Nondestructive control methods for evaluation of quality of large-size carbon products. Increase in efficiency and reliability of operation of aluminum electrolyzers: Collection of scientific papers VAMI, Leningrad: VAMI, 1987, 26-31 (in Russian)]

[4] Гребенкин А.Ф., Московенко И.Б. Акустический метод контроля качества углеродных изделий. Заводская лаборатория, 58 (2), 1992, 23-25 [Grebyonkin A.F., Moskovenko I.B. Acoustic method of quality control of carbon products. Plant laboratory, 58 (2), 1992, 23-25 (in Russian)] 